\title{
ELECTRICALLY CHARGED CONDUCTING DROPS REVISITED
}

\author{
BY \\ JAMES Q. FENG \\ Oak Ridge National Laboratory*, Oak Ridge, Tennessee
}

\begin{abstract}
Since its publication in 1882, Rayleigh's work on electrically charged conducting drops has been widely quoted, but its rigorous derivation has not been given in the literature. By means of the domain perturbation technique, this work presents a rigorous derivation of Rayleigh's results, following his approach with Lagrange's equation. With the systematic procedure, it becomes explicit that the first-order surface deformations result in a deviation of the drop surface potential of only second-order significance. Besides providing mathematical details, this work also reveals an apparent error in Rayleigh's original result for two-dimensional (cylindrical) drops.
\end{abstract}

1. Introduction. An electrically charged conducting drop is subjected to two opposing forces at its surface. One comes from the uniform surface tension, which tends to maintain the drop shape spherical or, in other words, to keep the surface area minimum for a given amount of liquid mass. The other force results from electrical repulsion from the charge, which tends to tear the drop apart. As the amount of charge increases to a critical value, the drop becomes unstable and disruption of the surface ensues. This phenomenon was first investigated in 1882 by Lord Rayleigh [1], who derived the characteristic frequencies of charged drop oscillations for both three-dimensional (spherical) and two-dimensional (cylindrical) configurations and established the amount of charge necessary to induce disruption of the drop surface. Like many of his works, Rayleigh's results for charged drops have been widely quoted in the literature and have found many important applications. Although the physical phenomena associated with such charged drops are generally regarded as well known, serious students as well as researchers were often frustrated by the overabundance of omitted steps in Rayleigh's original work. To alleviate this awkward situation, Hendricks and Schneider [2] provided a detailed derivation for a nearly spherical drop in 1963. Although their final results are correct, their derivation was not presented in a rigorous mathematical fashion with clear tracking of terms

Received October 31, 1994.

1991 Mathematics Subject Classification. Primary 35Q35, 41A60, 76B45, 78A30.

*Managed by Martin Marietta Energy Systems, Inc., under contract DE-AC05-84OR21400 with the U.

S. Department of Energy. The U. S. Government retains a nonexclusive, royalty-free license to publish or reproduce the published form of this contribution, or allow others to do so, for U. S. Government Purposes. 
representing the same order of magnitude. Also, their work was restricted to axisymmetric disturbances for three-dimensional drops. Nevertheless, because Hendricks and Schneider provided the only available derivation details found in the literature, their paper, together with Rayleigh's original work, has been appreciated and frequently quoted by many authors.

In view of its scientific significance, Rayleigh's results for charged drops deserve to be revisited with a rigorous mathematical treatment so that serious students and researchers can find in the literature the derivation details within the framework of a systematic procedure. By following Rayleigh's original approach, an expression for electrostatic energy due to the surface charge distribution should be derived based on the expression obtained for the electrical potential. Together with the expressions for the potential energy due to surface tension and the kinetic energy of the inviscid fluid motion, a Lagrangian can be written out. Then, going through standard operations, one can arrive at Rayleigh's results. In doing so, one must carry out the perturbation solutions to the second order because those potential energy terms are expected to be quadratic functions of the surface deformation amplitude. The ambiguous aspect in Hendricks and Schneider's derivation arises from the fact that in some quantities surface deformations were kept up to the quadratic (second-order) terms whereas in others only the linear (first-order) terms were retained. As is shown in this work, the domain perturbation procedure provides a mathematically rigorous derivation that was absent in the previous publications.

2. Domain perturbation technique. When analyzing a problem with the domain shape unknown a priori-often referred to as the free-boundary problem - it is convenient to map the domain of complicated unknown configuration onto a simple domain that is readily described in a standard curvilinear coordinate system. The domain perturbation technique was actually developed based on this concept. Although the prototype of this technique has appeared in the literature for many years, the formal expression was attributed to Joseph [3], who outlined a systematic procedure for calculating nonlinear effects from the higher-order problems by successive substitutions. More recently, this systematic procedure has been extended by Tsamopoulos and Brown [4] to include more than one free surface and by Feng [5] to include more than one small parameter. In the present problem of charged drops, however, we have only one free surface to deal with and need only one small parameter to describe the free surface deformations.

In general, the unknown shape of the free surface is uniquely described by a geometrical representation in three-dimensional space $\left(\zeta_{0}, \xi, \eta\right)$, e.g., the equation of the surface is written in Monge's form [6],

$$
\zeta_{0}=F(\xi, \eta)
$$

where $\zeta_{0}, \xi$, and $\eta$ can be taken as the three components in a curvilinear coordinate system. The problem domain enclosed (partially) by the complicated surface shape described by (2.1) can usually be transformed onto a domain with the corresponding surface shape simply described by $\zeta=1$, by introducing the change of coordinates

$$
\zeta_{0} \equiv \zeta F(\xi, \eta)
$$


With the small parameter $\varepsilon$ as a measure of the magnitude of the free surface deformations from the base-case shape $F(\xi, \eta)=1$, each dependent variable may be expanded in a Taylor series with respect to $\varepsilon$. For instance, an arbitrary function $f\left(\zeta_{0}, \xi, \eta ; \varepsilon\right)$ may be written in the form

$$
f\left(\zeta_{0}, \xi, \eta ; \varepsilon\right)=\sum_{n=0}^{\infty} \frac{\varepsilon^{n}}{n !} f^{[n]}(\zeta, \xi, \eta)
$$

where

$$
f^{\langle n\rangle}(\zeta, \xi, \eta) \equiv\left[\left(\frac{\partial}{\partial \varepsilon}+\zeta \frac{\partial F}{\partial \varepsilon} \frac{\partial}{\partial \zeta_{0}}\right)^{n} f\left(\zeta_{0}, \xi, \eta ; \varepsilon\right)\right]_{\substack{\xi=0 \\ \zeta_{0}=\zeta}} .
$$

Moreover, it is convenient to also use the notation

$$
f^{[n]}(\zeta, \xi, \eta) \equiv\left[\frac{\partial^{n}}{\partial \varepsilon^{n}} f\left(\zeta_{0}, \xi, \eta ; \varepsilon\right)\right]_{\substack{\varepsilon=0 \\ \zeta_{0}=\zeta}}
$$

because the expansion coefficient (2.4) can always be written in terms of (2.5), as described by Joseph [3]. In Rayleigh's original work [1], two particular equilibrium drop shapes were considered: a sphere (referred to as a three-dimensional drop hereafter) and a cylinder (referred to as a two-dimensional drop hereafter). Therefore, two particular curvilinear coordinate systems are convenient in the present work: the spherical system $(\zeta, \xi, \eta) \equiv(r, \theta, \phi)$ and the cylindrical system $(\zeta, \xi, \eta) \equiv(r, \phi, z)$. Like Rayleigh's original treatment [1], this work considers only translationally symmetric or two-dimensional deformations $(\partial / \partial z=0)$ for the case of a cylinder: consequently, the cylinder indeed corresponds to a drop in two-dimensional space.

3. Electric potential and electrostatic energy. One of the most significant omissions in Rayleigh's derivation [1] is the result for the electric potential at the deformed drop surface, which is needed for evaluating the electrostatic energy term in the Lagrangian. The electric potential $V$ in an insulating medium outside a conducting drop can be determined by solving Laplace's equation

$$
\nabla^{2} V=0 \quad\left(F \leq r_{0}<\infty\right)
$$

subjected to the following boundary conditions:

$$
\begin{gathered}
\nabla V \rightarrow 0 \quad \text { and } / \text { or } \quad V \rightarrow 0 \quad\left(r_{0} \rightarrow \infty\right) \\
\int(\mathbf{n} \cdot \nabla V)_{r_{0}=F} d S=-Q \\
\mathbf{n} \times \nabla V=0 \quad\left(r_{0}=F\right)
\end{gathered}
$$

where $\mathbf{n}$ is the unit normal vector at the free surface, $d S$ is the differential surface area, and $Q$ is the amount of electric charge on the drop. Equation (3.2) represents the condition for an electric field to vanish at a great distance from the drop bearing a finite amount of charge. It should be noted that the problem of a three-dimensional drop can be completely determined by imposing $V \rightarrow 0$ in (3.2) with $\nabla V \rightarrow 0$ then automatically 
satisfied, whereas the two-dimensional drop can satisfy $\nabla V \rightarrow 0$ but not $V \rightarrow 0$ in (3.2), because it involves an infinite source of electric charge in three-dimensional space. Therefore, in the problem of two-dimensional drops, an undetermined constant of integration is usually set to zero for the sake of complete mathematical description. Equation (3.3) guarantees the conservation of electric charge in the conducting drop. Equation (3.4) is the requirement of the continuous tangential component of the electric field across the conducting drop surface.

In what follows, details are given mainly for three-dimensional drops. The results for two-dimensional drops can be obtained in a similar manner; details are not repeated to avoid redundance. For the three-dimensional drop, we have, in spherical coordinates,

$$
\mathbf{n}=\frac{F \mathbf{e}_{r}-(\partial F / \partial \theta) \mathbf{e}_{\theta}-(\operatorname{cosec} \theta \partial F / \partial \phi) \mathbf{e}_{\phi}}{\left[F^{2}+(\partial F / \partial \theta)^{2}+(\operatorname{cosec} \theta \partial F / \partial \phi)^{2}\right]^{1 / 2}}
$$

and

$$
d S=\left[F^{2}+(\partial F / \partial \theta)^{2}+(\operatorname{cosec} \theta \partial F / \partial \phi)^{2}\right]^{1 / 2} F \sin \theta d \theta d \phi
$$

Equations (3.1)-(3.6) are made dimensionless by measuring length in units of $R$ (the radius of the undeformed spherical drop with volume $\frac{4}{3} \pi R^{3}$ ), electric potential in units of $\left(\sigma R / \varepsilon_{m}\right)^{1 / 2}$, and electric charge in units of $\left(\varepsilon_{m} \sigma R^{3}\right)^{1 / 2}$, where $\sigma$ denotes the uniform surface tension and $\varepsilon_{m}$ the permittivity of the insulating medium surrounding the drop, with SI units being used. The surface of the drop is described by $R F(\theta, \phi)$, where $F(\theta, \phi)$ is the dimensionless shape function of the drop. To complete the mathematical description, the shape function $F(\theta, \phi)$ is also subjected to two overall constraints, i.e., the requirement of the conservation of the drop volume,

$$
\int_{0}^{2 \pi} \int_{0}^{\pi} F^{3}(\theta, \phi) \sin \theta d \theta d \phi=4 \pi
$$

and the requirement that the center of mass of the drop remain fixed at the coordinate origin,

$$
\int_{0}^{2 \pi} \int_{0}^{\pi} F^{4}(\theta, \phi) \cos \theta \sin \theta d \theta d \phi=\int_{0}^{2 \pi} \int_{0}^{\pi} F^{4}(\theta, \phi) \sin ^{2} \theta e^{ \pm i \phi} d \theta d \phi=0
$$

The goal here is to determine the variation of the constant electric potential in the conducting drop surface for a given drop shape deformation. Following the domain perturbation procedure to expand (3.1)-(3.4), the zeroth-order solution that represents the undeformed spherical drop takes the simple form

$$
F^{\langle 0\rangle}=1 \quad \text { and } \quad V^{\langle 0\rangle}=\frac{Q}{4 \pi r}
$$


The first-order problem is governed by

$$
\begin{gathered}
\nabla^{2} V^{\langle 1\rangle}=0 \quad(1 \leq r<\infty), \\
V^{\langle 1\rangle} \rightarrow 0 \quad(r \rightarrow \infty), \\
\int_{0}^{2 \pi} \int_{0}^{\pi}\left(V_{r}^{\langle 1\rangle}+2 F^{\langle 1\rangle} V_{r}^{\langle 0\rangle}+F^{\langle 1\rangle} V_{r r}^{\langle 0\rangle}\right)_{r=1} \sin \theta d \theta d \phi=0, \\
\frac{\partial}{\partial \theta}\left(V^{\langle 1\rangle}+F^{\langle 1\rangle} V_{r}^{\langle 0\rangle}\right)=\frac{\partial}{\partial \phi}\left(V^{\langle 1\rangle}+F^{\langle 1\rangle} V_{r}^{\langle 0\rangle}\right)=0 \quad(r=1), \\
\int_{0}^{2 \pi} \int_{0}^{\pi} F^{\langle 1\rangle} \sin \theta d \theta d \phi=0, \\
\int_{0}^{2 \pi} \int_{0}^{\pi} F^{\langle 1\rangle} \cos \theta \sin \theta d \theta d \phi=\int_{0}^{2 \pi} \int_{0}^{\pi} F^{\langle 1\rangle} \sin ^{2} \theta e^{ \pm i \phi} d \theta d \phi=0 .
\end{gathered}
$$

For convenience, we may express $F^{\langle 1\rangle}$ and $V^{\langle 1\rangle}$ in terms of spherical harmonics $Y_{l k}(\theta, \phi)$, i.e.,

$$
F^{\langle 1\rangle}=\sum_{l=0}^{\infty} \sum_{k=-l}^{l} \alpha_{l k}^{\langle 1\rangle} Y_{l k}(\theta, \phi)
$$

and

$$
V^{\langle 1\rangle}=\sum_{l=0}^{\infty} \sum_{k=-l}^{l} \xi_{l k}^{\langle 1\rangle} r^{-l-1} Y_{l k}(\theta, \phi) .
$$

Here, $Y_{l k}(\theta, \phi)$ are normalized functions, i.e., $\left\langle Y_{l k}, Y_{l^{\prime} k^{\prime}}^{*}\right\rangle=\delta_{l l^{\prime}} \delta_{k k^{\prime}}$, and the symbol $\langle$, stands for the inner product with respect to $\theta$ and $\phi$ of the functions weighted with $\sin \theta$ on the interval $[\theta=0, \theta=\pi]$ and $[\phi=0, \phi=2 \pi]$, where $Y_{l k}^{*}=Y_{l,-k}$ denotes the complex conjugate of $Y_{l k}$. The results are then found to be

$$
\xi_{l k}^{\langle 1\rangle}=\frac{Q}{4 \pi} \alpha_{l k}^{\langle 1\rangle}(l \neq 0) \quad \text { and } \quad \xi_{00}^{\langle 1\rangle}=\alpha_{00}^{\langle 1\rangle}=\alpha_{1 k}^{\langle 1\rangle}=0
$$

Thus, the first-order deviation in the constant electric potential at the conducting drop surface is

$$
\begin{aligned}
V_{r=1}^{[1]} & \equiv\left(V^{\langle 1\rangle}+r F^{\langle 1\rangle} V_{r}^{\langle 0\rangle}\right)_{r=1} \\
& =\left[\int_{0}^{2 \pi} \int_{0}^{\pi}\left(V^{\langle 1\rangle}+r F^{\langle 1\rangle} V_{r}^{\langle 0\rangle}\right)_{r=1} Y_{00} \sin \theta d \theta d \phi\right] Y_{00} \\
& =\frac{1}{4 \pi}\left(\xi_{00}^{\langle 1\rangle}-\frac{Q}{4 \pi} \alpha_{00}^{\langle 1\rangle}\right)=0 .
\end{aligned}
$$

The equivalent integral form in (3.18) comes from the fact that Eq. (3.13) forces all the terms associated with spherical harmonics other than $Y_{00}$ to vanish at $r=1$; therefore, only the inner product with $Y_{00}=(4 \pi)^{-1 / 2}$ has a chance to survive.

Noteworthy here is the observation that even though the drop shape deformations are of the first order and the first-order correction to electric potential is nontrivial 
in general, the first-order deviation in the electric potential vanishes right at the drop surface. Therefore, the second-order problem must be considered to obtain the leadingorder deviation in the constant electric potential at the conducting drop surface that is needed for evaluating the electrostatic energy associated with the drop deformations. The second-order problem is governed by

$$
\begin{gathered}
\nabla^{2} V^{\langle 2\rangle}=0 \quad(1 \leq r<\infty), \\
V^{\langle 2\rangle} \rightarrow 0 \quad(r \rightarrow \infty), \\
\int_{0}^{2 \pi} \int_{0}^{\pi}\left(V_{r}^{\langle 2\rangle}+4 F^{\langle 1\rangle} V_{r}^{\langle 1\rangle}+2 F^{\langle 1\rangle} V_{r r}^{\langle 1\rangle}-2 F_{\theta}^{\langle 1\rangle} V_{\theta}^{\langle 1\rangle}-2 \frac{F_{\phi}^{\langle 1\rangle} V_{\phi}^{\langle 1\rangle}}{\sin ^{2} \theta}\right)_{r=1} \sin \theta d \theta d \phi=0, \\
\frac{\partial}{\partial \theta}\left(V^{\langle 2\rangle}+F^{\langle 2\rangle} V_{r}^{\langle 0\rangle}+2 F^{\langle 1\rangle} V_{r}^{\langle 1\rangle}+F^{\langle 1\rangle 2} V_{r r}^{\langle 0\rangle}\right) \\
=\frac{\partial}{\partial \phi}\left(V^{\langle 2\rangle}+F^{\langle 2\rangle} V_{r}^{\langle 0\rangle}+2 F^{\langle 1\rangle} V_{r}^{\langle 1\rangle}+F^{\langle 1\rangle 2} V_{r r}^{\langle 0\rangle}\right)=0 \\
\int_{0}^{2 \pi} \int_{0}^{\pi}\left(F^{\langle 2\rangle}+3 F^{\langle 1\rangle 2}\right) \cos \theta \sin \theta d \theta d \phi \\
\left.\int_{0}^{2 \pi} \int_{0}^{\pi}\left(F^{\langle 2\rangle}+3 F^{\langle 1\rangle 2}\right) \sin { }^{2} \theta e^{ \pm i \phi} d \theta d \phi=0.21\right),
\end{gathered}
$$

Like $F^{\langle 1\rangle}$ and $V^{\langle 1\rangle}$ in (3.16), $F^{\langle 2\rangle}$ and $V^{\langle 2\rangle}$ may be expressed in terms of normalized spherical harmonics $Y_{l k}(\theta, \phi)$. Thus, the second-order results are found to be

$$
\begin{aligned}
\xi_{00}^{\langle 2\rangle} & =0 \\
\xi_{l k}^{\langle 2\rangle} & =\frac{Q}{4 \pi}\left(\alpha_{l k}^{\langle 2\rangle}+\sum_{l^{\prime}} \sum_{k^{\prime}} \sum_{l^{\prime \prime}} \sum_{k^{\prime \prime}} 2 l^{\prime \prime} \alpha_{l^{\prime} k^{\prime}}^{\langle 1\rangle} \alpha_{l^{\prime \prime} k^{\prime \prime}}^{\langle 1\rangle}\left\langle Y_{l^{\prime} k^{\prime}} Y_{l^{\prime \prime} k^{\prime \prime}}, Y_{l k}^{*}\right\rangle\right) \quad(l \neq 0) \\
\alpha_{00}^{\langle 2\rangle} & =-2 \sum_{l} \sum_{k} \alpha_{l k}^{\langle 1\rangle} \alpha_{l,-k}^{\langle 1\rangle}, \\
\alpha_{1 k}^{\langle 2\rangle} & =-3 \sum_{l^{\prime}} \sum_{k^{\prime}} \sum_{l^{\prime \prime}} \sum_{k^{\prime \prime}} \alpha_{l^{\prime} k^{\prime}}^{\langle 1\rangle} \alpha_{l^{\prime \prime} k^{\prime \prime}}^{\langle 1\rangle}\left\langle Y_{l^{\prime} k^{\prime}} Y_{l^{\prime \prime} k^{\prime \prime}}, Y_{1 k}^{*}\right\rangle
\end{aligned}
$$

Because

$$
V^{[2]} \equiv V^{\langle 2\rangle}+r F^{\langle 2\rangle} V_{r}^{\langle 0\rangle}+2 r F^{\langle 1\rangle} V_{r}^{\langle 1\rangle}+r^{2} F^{\langle 1\rangle 2} V_{r r}^{\langle 0\rangle}
$$


Eq. (3.22) as satisfied by (3.25b) implies that at the drop surface [see discussion following $(3.18)]$,

$$
\begin{aligned}
V_{r=1}^{[2]} & =\left[\int_{0}^{2 \pi} \int_{0}^{\pi}\left(V^{\langle 2\rangle}+F^{\langle 2\rangle} V_{r}^{\langle 0\rangle}+2 F^{\langle 1\rangle} V_{r}^{\langle 1\rangle}+F^{\langle 1\rangle 2} V_{r r}^{\langle 0\rangle}\right)_{r=1} Y_{00} \sin \theta d \theta d \phi\right] Y_{00} \\
& =\frac{1}{4 \pi}\left[\xi_{00}^{\langle 2\rangle}-\frac{Q}{4 \pi} \alpha_{00}^{\langle 2\rangle}-2 \frac{Q}{4 \pi} \sum_{l=2}^{\infty} \sum_{k=-l}^{l}(l+1) \alpha_{l k}^{\langle 1\rangle} \alpha_{l,-k}^{\langle 1\rangle}+2 \frac{Q}{4 \pi} \sum_{l=2}^{\infty} \sum_{k=-l}^{l} \alpha_{l k}^{\langle 1\rangle} \alpha_{l,-k}^{\langle 1\rangle}\right] \\
& =-2 \frac{Q}{(4 \pi)^{2}} \sum_{l=2}^{\infty} \sum_{k=-l}^{l}(l-1) \alpha_{l k}^{\langle 1\rangle} \alpha_{l,-k}^{\langle 1\rangle} .
\end{aligned}
$$

Hence, the surface potential of a conducting drop with the leading-order correction is given by

$$
V_{r=1} \approx\left(V^{[0]}+\frac{\varepsilon^{2}}{2} V^{[2]}\right)_{r=1}=\frac{Q}{(4 \pi)^{2}}\left[1-\varepsilon^{2} \sum_{l=2}^{\infty} \sum_{k=-l}^{l}(l-1) \alpha_{l k}^{\langle 1\rangle} \alpha_{l,-k}^{\langle 1\rangle}\right] .
$$

Because the electric charge appears only at the conducting surface that is at a constant potential, the electrostatic energy of the system (see, e.g., [7]) evaluated from the sphere of equilibrium is then given by

$$
W \equiv \frac{1}{2} Q\left(V-V^{[0]}\right)_{r=1} \approx-\frac{1}{2}\left(\frac{Q}{4 \pi}\right)^{2} \varepsilon^{2} \sum_{l=2}^{\infty} \sum_{k=-l}^{l}(l-1) \alpha_{l k}^{\langle 1\rangle} \alpha_{l,-k}^{\langle 1\rangle} .
$$

In general, the second-order results for $V^{\langle 2\rangle}$ and also $V^{[2]}$ can be expressed in terms of $F^{\langle 2\rangle}$ as well as $F^{\langle 1\rangle}, V^{\langle 1\rangle}$, and $V^{\langle 0\rangle}$, as indicated in (3.25) and (3.26). The shape function $\left(F^{\langle 1\rangle}, F^{\langle 2\rangle}, \ldots\right)$ cannot be completely determined by the equation set given so far and is treated as a given function. However, it may be determined when the physical mechanisms of drop deformations are described and included in the mathematical system, e.g., as presented later in this paper for the situation when the drop is undergoing oscillations.

Particularly for a conducting drop, the electrostatic energy of the system is completely determined by the constant potential at the drop surface. It turns out that the contribution from the second-order solution to the constant surface potential comes only from $\alpha_{00}^{\langle 2\rangle}$ [see (3.27)], which can be written in terms of $\alpha_{l k}^{\langle 1\rangle}$ [as in (3.25c)] by virtue of the overall constraint (3.23). Hence, the final result of the leading-order deviation in the constant surface potential of a deformed drop (3.28) is completely expressed in terms of the first-order drop shape deformations $\alpha_{l k}^{\langle 1\rangle}$. This might be why without rigorously considering the second-order problem, Hendricks and Schneider [2] could still obtain the correct final result.

For a two-dimensional drop of a volume $\pi R^{2}$ per unit length with its surface described by $R F(\phi)$, where $F(\phi)$ is the dimensionless drop shape function in cylindrical coordinates (in polar plane only), Eqs. (3.5) and (3.6) may be replaced by

$$
\mathbf{n}=\frac{F \mathbf{e}_{r}-(\partial F / \partial \phi) \mathbf{e}_{\phi}}{\left[F^{2}+(\partial F / \partial \phi)^{2}\right]^{1 / 2}}
$$


and

$$
d S=\left[F^{2}+(\partial F / \partial \phi)^{2}\right]^{1 / 2} d \phi .
$$

The two overall constraints to the drop shape function (3.7) and (3.8) may then be replaced by

$$
\int_{0}^{2 \pi} F^{2}(\phi) d \phi=2 \pi
$$

and

$$
\int_{0}^{2 \pi} F^{3}(\phi) e^{ \pm i \phi} d \phi=0
$$

The zeroth-order solution that represents the undeformed cylindrical drop is found to be

$$
F^{\langle 0\rangle}=1 \quad \text { and } \quad V^{\langle 0\rangle}=-\frac{Q}{2 \pi} \log r
$$

which satisfies $\nabla V^{\langle 0\rangle} \rightarrow 0$, but not $V^{\langle 0\rangle} \rightarrow 0$, as $r \rightarrow \infty$, for the reason explained previously. Note that $Q$ here denotes the amount of electric charge per unit length and the undetermined constant of integration is always set to zero.

For higher-order problems, we may conveniently express $F^{\langle n\rangle}$ and $V^{\langle n\rangle}$ in harmonic forms, i.e.,

$$
F^{\langle n\rangle}=\sum_{l=0}^{\infty}\left(\alpha_{l}^{\langle n\rangle} e^{i l \phi}+\alpha_{-l}^{\langle n\rangle} e^{-i l \phi}\right)
$$

and

$$
V^{\langle n\rangle}=\zeta_{0}^{\langle n\rangle} \log r+\sum_{l=1}^{\infty} r^{-l}\left(\xi_{l}^{\langle n\rangle} e^{i l \phi}+\xi_{-l}^{\langle n\rangle} e^{-i l \phi}\right) .
$$

With some corresponding modifications in the governing equations presented for the three-dimensional drop, the first-order and second-order results are obtained as

$$
\xi_{l}^{\langle 1\rangle}=\frac{Q}{2 \pi} \alpha_{l}^{\langle 1\rangle}(l \neq 0) \quad \text { and } \quad \zeta_{0}^{\langle 1\rangle}=\alpha_{0}^{\langle 1\rangle}=\alpha_{ \pm 1}^{\langle 1\rangle}=0,
$$

and

$$
\zeta_{0}^{\langle 2\rangle}=0, \quad \alpha_{0}^{\langle 2\rangle}=-2 \sum_{l=2}^{\infty} \alpha_{l}^{\langle 1\rangle} \alpha_{-l}^{\langle 1\rangle}, \text { etc. }
$$

Hence, the first-order and second-order deviations in the constant electric potential at the conducting drop surface are given by

$$
V_{r=1}^{[1]}=\left(V^{\langle 1\rangle}+r F^{\langle 1\rangle} V_{r}^{\langle 0\rangle}\right)_{r=1}=0
$$

and

$$
V_{r=1}^{[2]}=-4 \frac{Q}{2 \pi} \sum_{l=2}^{\infty}(l-1) \alpha_{l}^{\langle 1\rangle} \alpha_{-l}^{\langle 1\rangle} .
$$

Thus, the electrostatic energy of the two-dimensional drop is found to be

$$
W \equiv \frac{1}{2} Q\left(V-V^{[0]}\right)_{r=1} \approx-\frac{Q^{2}}{2 \pi} \varepsilon^{2} \sum_{l=2}^{\infty}(l-1) \alpha_{l}^{\langle 1\rangle} \alpha_{-l}^{\langle 1\rangle} .
$$


4. Surface energy and kinetic energy. Although Rayleigh also omitted steps in obtaining the surface energy and kinetic energy in his original work [1], these details can be found in many places and are also quite easy to derive. Nonetheless, a brief derivation is given here for the sake of completeness and consistency of notation.

For the three-dimensional drop, the dimensionless surface energy evaluated from its equilibrium shape is [see (3.6)]

$$
\begin{aligned}
S & =\int_{0}^{2 \pi} \int_{0}^{\pi}\left[F^{2}+(\partial F / \partial \theta)^{2}+(\operatorname{cosec} \theta \partial F / \partial \phi)^{2}\right]^{1 / 2} F \sin \theta d \theta d \phi-4 \pi \\
& \approx \frac{\varepsilon^{2}}{2} \int_{0}^{2 \pi} \int_{0}^{\pi}\left[2 F^{\langle 1\rangle 2}+2 F^{\langle 2\rangle}+\left(\partial F^{\langle 1\rangle} / \partial \theta\right)^{2}+\left(\operatorname{cosec} \theta \partial F^{\langle 1\rangle} / \partial \phi\right)^{2}\right] \sin \theta d \theta d \phi \\
& =\frac{\varepsilon^{2}}{2} \sum_{l=2}^{\infty} \sum_{k=-l}^{l}(l-1)(l+2) \alpha_{l k}^{\langle 1\rangle} \alpha_{l,-k}^{\langle 1\rangle},
\end{aligned}
$$

whereas for the two-dimensional drop, we have from (3.31)

$$
\begin{aligned}
S & =\int_{0}^{2 \pi}\left[F^{2}+(\partial F / \partial \phi)^{2}\right]^{1 / 2} d \phi-2 \pi \approx \frac{\varepsilon^{2}}{2} \int_{0}^{2 \pi}\left[F^{\langle 2\rangle}+\left(\partial F^{\langle 1\rangle} / \partial \phi\right)^{2}\right] d \phi \\
& =2 \pi \varepsilon^{2} \sum_{l=2}^{\infty}\left(l^{2}-1\right) \alpha_{l}^{\langle 1\rangle} \alpha_{-l}^{\langle 1\rangle} .
\end{aligned}
$$

Apparently, the second-order shape deformation $F^{\langle 2\rangle}$ must be included in the calculation of surface areas. Because of the nature of the integral, however, only $\alpha_{00}^{\langle 2\rangle}$ (or $\alpha_{0}^{\langle 2\rangle}$ ) is needed. Thus the surface energy term is completely expressed in terms of $\alpha_{l k}^{\langle 1\rangle}$ (or $\alpha_{l}^{\langle 1\rangle}$ ) through (3.25c) [or (3.37b)].

The dimensionless kinetic energy of a fluid drop in a tenuous medium takes the nontrivial leading-order form

$$
\begin{aligned}
\int \frac{1}{2} u^{2} d \mathcal{V} & =\frac{1}{2} \int(\Phi \mathbf{n} \cdot \nabla \Phi)_{r_{0}=F} d S \\
& \approx \frac{1}{2} \varepsilon^{2} \int\left(\Phi^{\langle 1\rangle} \frac{\partial \Phi^{\langle 1\rangle}}{\partial r}\right)_{r=1} d S
\end{aligned}
$$

where the relation of perturbation velocity $u=\nabla \Phi$ is used by assuming the flow field to be irrotational and solenoidal, an assumption that is usually reasonable for the situations where viscous effects are negligible and the fluid is incompressible. The nondimensionalization here is achieved by measuring the velocity potential in units of $(\sigma R / \rho)^{1 / 2}$, with $\rho$ denoting the fluid density in the drop. The dimensionless velocity potential $\Phi$ is governed by Laplace's equation in the region $r \leq 1$. Because the zeroth-order velocity potential is set to zero, there is no need to consider the second-order solution for the velocity potential to evaluate (4.3). Hence, we have the nontrivial leading-order solutions

$$
\Phi^{\langle 1\rangle}=\sum_{l=1}^{\infty} \sum_{k=-l}^{l} \beta_{l k}^{\langle 1\rangle} r^{l} Y_{l k}(\theta, \phi)
$$


and

$$
\Phi^{\langle 1\rangle}=\sum_{l=1}^{\infty} r^{l}\left(\beta_{l}^{\langle 1\rangle} e^{i l \phi}+\beta_{-l}^{\langle 1\rangle} e^{-i l \phi}\right)
$$

for the three-dimensional drop and two-dimensional drop, respectively. Moreover, to the first order, the kinematic condition relates the velocity potential to the drop shape function as

$$
\frac{\partial \Phi^{\langle 1\rangle}}{\partial r}=\frac{\partial F^{\langle 1\rangle}}{\partial t},
$$

where the dimensionless time $t$ is obtained by measuring time in units of $\left(\rho R^{3} / \sigma\right)^{1 / 2}$. Thus, for the three-dimensional drop, the leading-order kinetic energy is given by

$$
K \approx \frac{1}{2} \varepsilon^{2} \sum_{l=2}^{\infty} \sum_{k=-l}^{l} \frac{1}{l} \frac{d \alpha_{l k}^{\langle 1\rangle}}{d t} \frac{d \alpha_{l,-k}^{\langle 1\rangle}}{d t}
$$

and for the two-dimensional drop,

$$
K \approx 2 \pi \varepsilon^{2} \sum_{l=2}^{\infty} \frac{1}{l} \frac{d \alpha_{l}^{\langle 1\rangle}}{d t} \frac{d \alpha_{-l}^{\langle 1\rangle}}{d t} .
$$

5. Lagrangian and characteristic frequency. The Lagrangian for the problem can be written in terms of the kinetic energy, electrostatic energy, and surface energy as

$$
L \equiv K-W-S .
$$

Hence, (4.6), (4.1), and (3.29) yield

$$
L=\frac{1}{2} \varepsilon^{2} \sum_{l=2}^{\infty} \sum_{k=-l}^{l}\left[\frac{1}{l} \frac{d \alpha_{l k}^{\langle 1\rangle}}{d t} \frac{d \alpha_{l,-k}^{\langle 1\rangle}}{d t}+\left(\frac{Q}{4 \pi}\right)^{2}(l-1) \alpha_{l k}^{\langle 1\rangle} \alpha_{l,-k}^{\langle 1\rangle}-(l-1)(l+2) \alpha_{l k}^{\langle 1\rangle} \alpha_{l,-k}^{\langle 1\rangle}\right]
$$

for the three-dimensional drop, and (4.7), (4.2), and (3.40) yield

$$
L=2 \pi \varepsilon^{2} \sum_{l=2}^{\infty}\left[\frac{1}{l} \frac{d \alpha_{l}^{\langle 1\rangle}}{d t} \frac{d \alpha_{-l}^{\langle 1\rangle}}{d t}+\left(\frac{Q}{2 \pi}\right)^{2}(l-1) \alpha_{l}^{\langle 1\rangle} \alpha_{-l}^{\langle 1\rangle}-\left(l^{2}-1\right) \alpha_{l}^{\langle 1\rangle} \alpha_{-l}^{\langle 1\rangle}\right]
$$

for the two-dimensional drop. Then Lagrange's equations,

$$
\frac{d}{d t} \frac{\partial L}{\partial\left(d \alpha_{l,-k}^{\langle 1\rangle} / d t\right)}-\frac{\partial L}{\partial \alpha_{l,-k}^{\langle 1\rangle}}=0 \text { (for three-dimensional drops) }
$$

and

$$
\frac{d}{d t} \frac{\partial L}{\partial\left(d \alpha_{-l}^{\langle 1\rangle} / d t\right)}-\frac{\partial L}{\partial \alpha_{-l}^{\langle 1\rangle}}=0 \text { (for two-dimensional drops) }
$$

lead to

$$
\frac{d^{2} \alpha_{l k}^{\langle 1\rangle}}{d t^{2}}+l(l-1)(l+2)\left[1-\frac{1}{l+2}\left(\frac{Q}{4 \pi}\right)^{2}\right] \alpha_{l k}^{\langle 1\rangle}=0
$$


and

$$
\frac{d^{2} \alpha_{l}^{\langle 1\rangle}}{d t^{2}}+l\left(l^{2}-1\right)\left[1-\frac{1}{l+1}\left(\frac{Q}{2 \pi}\right)^{2}\right] \alpha_{l}^{\langle 1\rangle}=0
$$

Finally, we obtain the characteristic frequency $\omega_{l k}$ from (5.6) for three-dimensional drops as

$$
\omega_{l k}^{2}=l(l-1)(l+2)\left[1-\frac{1}{l+2}\left(\frac{Q}{4 \pi}\right)^{2}\right]
$$

and $\omega_{l}$ from (5.7) for two-dimensional drops as

$$
\omega_{l}^{2}=l\left(l^{2}-1\right)\left[1-\frac{1}{l+1}\left(\frac{Q}{2 \pi}\right)^{2}\right]
$$

Converting back to the dimensional form and changing from the SI units used in this work to the Gaussian system of units used by Rayleigh, (5.8) becomes

$$
\omega_{l k}^{2}=\frac{\sigma l(l-1)(l+2)}{\rho R^{3}}\left[1-\frac{1}{l+2} \frac{Q^{* 2}}{4 \pi R^{3} \sigma}\right]
$$

whereas (5.9) yields

$$
\omega_{l}^{2}=\frac{\sigma l\left(l^{2}-1\right)}{\rho R^{3}}\left[1-\frac{1}{l+1} \frac{Q^{* 2}}{\pi L^{2} R \sigma}\right],
$$

where $Q^{*}$ denotes the electric charge in "electrostatic units" (or esu). Thus, the condition for a spherical (three-dimensional) drop to be stable is $\sigma>Q^{* 2} /\left(16 \pi R^{3}\right)$ and that for a cylindrical (two-dimensional) drop to be stable with respect to the translationally symmetric disturbances is $\sigma>Q^{* 2} /\left(3 \pi L^{2} R\right)$. It should be noted that, for the sake of easy comparison, all the quantities here associated with (5.10) and (5.11) are dimensional in Gaussian units and in (5.11) $Q^{*}$ represents the amount of electric charge on length $L$ of the cylinder, as Rayleigh defined in his original work. Rayleigh's result for threedimensional drops is identical to that given by (5.10), whereas his original result for two-dimensional drops takes a form slightly different from (5.11) as

$$
\omega_{l}^{2}=\frac{\sigma l\left(l^{2}-1\right)}{\rho R^{3}}\left[1-\frac{2}{l+1} \frac{Q^{* 2}}{\pi L^{2} R \sigma}\right]
$$

which leads to a slightly different condition for stability: $\sigma>2 Q^{* 2} /\left(3 \pi L^{2} R\right)$.

To verify the present result (5.11), traditional derivation (e.g., [5], [8], [9]) for twodimensional drops has also been conducted, which actually involves only a first-order solution whereby the derivation is less complicated. The result has been found identical to (5.11). Apparently, Rayleigh invoked an error in his original work [1] for the case of two-dimensional drops. 
6. Discussion. Rayleigh's approach of formulating the Lagrangian and then solving Lagrange's equation for the characteristic frequency of charged conducting drops requires that the second-order problems be evaluated. Because of complex nonlinearities, systematic tracking of terms of the same order of magnitude is desirable to avoid derivation errors. In this work, rigorous derivation of Rayleigh's results on charged drops [1] is facilitated by means of the domain-perturbation technique [3]. Although the final results for the stability condition can also be obtained by a first-order perturbation analysis (e.g., [8], [9]), evaluating the second-order problems needed for solving Lagrange's equation provides a quantitative relationship between the deviation of the constant potential at the conducting drop surface and the drop surface deformations. An explicit conclusion can thereby be drawn that the first-order surface deformations result in a deviation magnitude of the drop surface potential having only second-order significance.

Acknowledgment. This work was partially supported by the Division of Engineering and Geosciences, the Office of Basic Energy Sciences, and the U. S. Department of Energy, under contract DE-AC05-84OR21400 with Martin Marietta Energy Systems, Inc.

\section{REFERENCES}

[1] Lord Rayleigh, On the equilibrium of liquid conducting masses charged with electricity, Philos. Mag. 5 (14), 184-186 (1882)

[2] C. D. Hendricks and J. M. Schneider, Stability of a conducting droplet under the influence of surface tension and electrostatic forces, Amer. J. Phys. 31, 450-453 (1963)

[3] D. D. Joseph, Domain perturbations: The higher order theory of infinitesimal water waves, Arch. Rational Mech. Anal. 51, 294-303 (1973)

[4] J. A. Tsamopoulos and R. A. Brown, Dynamic centering of liquid shells, Phys. Fluids 30, 27-35 (1987)

[5] J. Q. Feng, A method of multiple-parameter perturbations with an application to drop oscillations in an electric field, Quart. Appl. Math. 48, 555-567 (1990)

[6] C. E. Weatherburn, Differential Geometry of Three Dimensions, Cambridge University Press, 1927

[7] J. D. Jackson, Classical Electrodynamics, John Wiley and Sons, 1975

[8] J. A. Tsamopoulos and R. A. Brown, Resonant oscillations of inviscid charged drops, J. Fluid Mech. 147, 373-395 (1984)

[9] J. Q. Feng and K. V. Beard, Three-dimensional oscillation characteristics of electrostatically deformed drops, J. Fluid Mech. 227, 429-447 (1991) 